\title{
Calcium, vitamin $D$ and dairy intake in relation to type 2 diabetes risk in a Japanese cohort
}

\author{
K. Kirii • T. Mizoue $\cdot$ H. Iso • Y. Takahashi • M. Kato • \\ M. Inoue • M. Noda $\cdot$ S. Tsugane $\cdot$ for the Japan Public \\ Health Center-based Prospective Study Group
}

Received: 18 May 2009 / Accepted: 8 September 2009/Published online: 13 October 2009

(C) Springer-Verlag 2009

\begin{abstract}
Aims/hypothesis Calcium and vitamin D have been implicated in the development of type 2 diabetes, but epidemi-
\end{abstract}

Electronic supplementary material The online version of this article (doi:10.1007/s00125-009-1554-x) contains a list of the members of the Japan Public Health Center-based Prospective Study Group, which is available to authorised users.

\footnotetext{
K. Kirii $\cdot$ T. Mizoue

Department of Epidemiology and International Health, Research Institute, International Medical Center of Japan, Tokyo, Japan

K. Kirii

Department of Public Health Medicine, Graduate School of Comprehensive Medical Sciences, University of Tsukuba,

Tsukuba,

Ibaraki, Japan

H. Iso

Public Health, Department of Social and Environmental Medicine, Osaka University Graduate School of Medicine,

Suita, Osaka, Japan

Y. Takahashi $\cdot$ M. Noda $(\square)$

Department of Diabetes and Metabolic Medicine,

International Medical Center of Japan,

1-21-1 Toyama, Shinjuku-ku,

Tokyo 162-8655, Japan

e-mail:mnoda@imcj.hosp.go.jp

M. Kato

Japan Foundation for the Promotion of International Medical

Research Cooperation,

Shinjuku-ku, Tokyo, Japan

$\mathrm{M}$. Inoue $\cdot \mathrm{S}$. Tsugane

Epidemiology and Prevention Division, Research Center for

Cancer Prevention and Screening, National Cancer Center,

Chuo-ku, Tokyo, Japan

ological evidence is limited. We examined prospectively the relation of calcium and vitamin $\mathrm{D}$ intake to type 2 diabetes risk in a Japanese cohort.

Methods Participants were 59,796 middle-aged and older men and women, who participated in the Japan Public Health Center-based Prospective Study and had no history of type 2 diabetes or other serious diseases. Dietary intake of calcium and vitamin D were estimated using a validated food frequency questionnaire. Logistic regression was used to assess the association between intake of these nutrients and self-reported newly diagnosed type 2 diabetes.

Results During a 5 year follow-up, 1,114 cases of type 2 diabetes were documented. Overall, calcium intake was not associated with a significantly lower risk of type 2 diabetes; the multivariable odds ratio for the highest vs lowest quartiles was 0.93 (95\% CI $0.71-1.22)$ in men and 0.76 (95\% CI 0.56-1.03) in women. However, among participants with a higher vitamin $\mathrm{D}$ intake, calcium intake was inversely associated with diabetes risk; the odds ratio for the highest vs lowest intake categories was $0.62(95 \% \mathrm{CI}$ $0.41-0.94)$ in men and 0.59 (95\% CI $0.38-0.91)$ in women. Dairy food intake was significantly associated with a lower risk of type 2 diabetes in women only.

Conclusions/interpretation Calcium and vitamin D may not be independently associated with type 2 diabetes risk. Our finding suggesting a joint action of these nutrients against type 2 diabetes warrants further investigation.

Keywords Calcium · Cohort studies · Type 2 diabetes Vitamin D

Abbreviations
$\begin{array}{ll}\text { 25-OHD } & \text { 25-Hydroxyvitamin D } \\ \text { JPHC } & \text { Japan Public Health Center-based } \\ & \text { Prospective Study }\end{array}$




\section{Introduction}

The number of people with type 2 diabetes has been increasing worldwide, with an estimated prevalence of $2.8 \%$ in 2000 and $4.4 \%$ in 2030 [1]. According to community-based studies, the prevalence of diabetes in Japan has rapidly increased during the past two decades [2]. Insight into the role of dietary factors in the development of diabetes may contribute to its prevention.

In experimental studies, calcium and vitamin D have been shown to improve pancreatic beta cell function and peripheral insulin sensitivity [3-5]. In humans, evidence on this issue so far is mainly derived from cross-sectional studies as reviewed [6], while findings from prospective studies are limited and inconsistent. Two cohort studies found a moderate, but not statistically significant association between dietary calcium intake and the risk of diabetes after adjustments for other dietary factors [7, 8], although the association with supplemental intake was statistically significant. A high intake of dairy foods, a major food source of calcium, has also been shown to be associated with a lower risk of type 2 diabetes [7-10]. Similarly, vitamin D intake from supplement [7] or supplement plus diet [11], but not from diet alone was associated with a lower incidence of diabetes. As regards blood vitamin D, a prospective study in Finland showed an inverse association between serum 25-hydroxyvitamin D (25-OHD) concentrations and the risk of type 2 diabetes [12]. However, in a large-scale randomised controlled trial in US American women [13], calcium plus vitamin $\mathrm{D}_{3}$ supplementation did not reduce the risk of developing diabetes over 7 years of follow-ups. Given the scarcity and inconsistency of prospective evidence [6], it is unclear whether these nutrients prevent type 2 diabetes.

To the best of our knowledge, there are no reports on the relation between both calcium and vitamin $\mathrm{D}$ intake, and the incidence of type 2 diabetes in the Japanese population, which consumes, on average, a relatively low amount of calcium [14]. We therefore examined the associations of calcium, vitamin $\mathrm{D}$ and dairy foods intake with the risk of type 2 diabetes in a large-scale cohort of the Japanese population. Since calcium and vitamin D have been hypothesised to act jointly, rather than independently, in reducing the risk of diabetes [7], we also explored their combined effect on the risk of type 2 diabetes.

\section{Methods}

Study cohort The Japan Public Health Center-based Prospective Study (JPHC) was established in 1990 for cohort I and in 1993 for cohort II. Details of the study design have been described elsewhere [15]. The participants of cohort I included residents, aged 40 to 59 years, in five Japanese Public Health Center areas (Iwate, Akita, Nagano, Okinawa and Tokyo); the participants of cohort II included residents, aged 40 to 69 years, in six Public Health Center areas (Ibaraki, Niigata, Kouchi, Nagasaki, Okinawa and Osaka). Study participants were informed about the objectives of the study and those who responded to the survey questionnaire were regarded as consenting to participate in the study. This study was approved by the institutional review board of the National Cancer Center, Tokyo, Japan.

Questionnaire surveys A questionnaire survey was conducted at baseline and at the 5- and 10-year follow-ups. Information on medical history and health-related lifestyles, including smoking, drinking and dietary habits, was obtained in each survey. In the present analysis, we used data from the 5-year survey, which was carried out in 1995 for cohort I and in 1998 for cohort II, as baseline. We did this because the questionnaire used for that survey contained more comprehensive information on food intake than that used for the first survey.

Participants Within the study population at baseline $(n=$ 140,420), we excluded residents of two areas (Tokyo and Osaka) because of the differences in recruitment criteria. Of the remaining 116,672 individuals, $95,373(82 \%)$ responded to the baseline survey. Of these, 80,128 (84\%) completed a questionnaire at the 5-year survey, which provided the baseline of the present analysis. Of these, 71,075 (89\%) responded to a questionnaire at the 10-year follow-up. We excluded individuals with a history of stroke, cardiovascular disease, cancer, chronic liver disease or kidney disease $(n=$ $10,694)$ at the first and 5-year follow-up surveys. We also excluded those with $>3$ and $<3$ standard deviations of energy intake $(n=585)$. As a result, 59,796 individuals $(25,877$ men, 33,919 women) were analysed.

Food frequency questionnaire The food frequency questionnaire used for the 5-year follow-up survey included questions about 147 food and beverage items with standard portions/units and eating frequency. Nine response options were available for eating frequency: rarely, one to two times/month, 1 to 2 days/week, 3 to 4 days/week, 5 to 6 days/week, once a day, two to three times/day, four to six times/day and $\geq 7$ times/day. Slightly different options were used for beverage intake: rarely, 1 to 2 days/week, 3 to 4 days/week, 5 to 6 days/week, once a day, two to three times/day, four to six times/day, seven to nine times/day and $\geq 10$ times/day. A standard portion size was specified for each food item and the respondents were asked to choose their usual portion from among three categories: less than half, same and more than 1.5 times the standard portion. We calculated the average daily intake of nutrients, 
including calcium and vitamin $\mathrm{D}$, by multiplying the frequency of the consumption of each food by its nutrient content per serving and totalling the nutrient intake for all food items. In Japan, vitamin D fortification of dairy products was not a common practice at the time of survey. The validity of the dietary calcium and vitamin D intake was assessed in a sub-sample of the cohort by comparing the estimated intake according to the questionnaire with that based on dietary records [16]. The Spearman's correlation coefficients of the energy-adjusted intake of calcium and vitamin D between the questionnaire and the dietary records were 0.54 and 0.77 for the men in cohort I, 0.68 and 0.56 for the men in cohort II, 0.45 and 0.43 for the women in cohort I and 0.68 and 0.52 for the women in cohort II, respectively [16].

Ascertainment of diabetes mellitus In the 10-year follow-up questionnaire, study participants were asked if they had ever been diagnosed as having diabetes and, if so, when the initial diagnosis had been made. Because the 5-year survey was used as baseline in the present study, only participants who were subsequently diagnosed (i.e. after 1995 for cohort I and after 1998 for cohort II) were regarded as incident cases during the follow-up. We did not obtain information on the type of diabetes; however, considering the minimum age of the study population ( 45 years old at baseline [5-year survey]), we reasonably assumed that most reported cases were type 2 diabetes. To assess the validity of self-reported diabetes, we examined a series of medical records of some study participants in three districts of the study areas, finding that $94 \%$ of the self-reported cases of diabetes were confirmed by medical records [17]. We also examined the sensitivity of self-reported diabetes among JPHC cohort I participants for whom data on plasma glucose were available from the 1990 health check-up. Of the 6,118 participants with plasma glucose data, 248 had self-reported diabetes. Of the 5,870 participants who did not have selfreported diabetes, 49 participants $(0.83 \%)$ had diabetes based on a single measurement and according to the diagnostic standards commonly used in Japan at that time (1990), i.e.: (1) fasting plasma glucose $\geq 7.8 \mathrm{mmol} / \mathrm{l}$; and (2) casual plasma glucose $\geq 11 \mathrm{mmol} / \mathrm{l}$ [18]. Taking into account the above-mentioned positive predictive value, the sensitivity and specificity of self-reported diabetes were $82.9 \%$ and $99.7 \%$, respectively.

Statistical analysis Analyses were performed on men and women, separately. Dietary intakes of calcium and vitamin D were adjusted for total energy intake using the residual method [19]. Baseline characteristics were presented according to quartiles of dietary calcium and vitamin D intake, and their trend associations assessed using a linear regression analysis for continuous variables or a logistic regression for categorical variables, with the median value of calcium or vitamin D intake in each category assigned to the corresponding category. A logistic regression analysis was used to assess the associations of the dietary intake of calcium, vitamin D and dairy food with the incidence of type 2 diabetes. The odds ratios and 95\% CIs were calculated for each quartile of intake using the lowest consumption category as a reference. All logistic regression analyses were adjusted for age (year; continuous) and study area (nine Public Health Centers). The multivariate analyses were additionally adjusted for the following nine factors: (1) BMI $\left(<21,21-22.9,23-24.9,25-26.9\right.$ or $\left.\geq 27 \mathrm{~kg} / \mathrm{m}^{2}\right)$; (2) family history of diabetes mellitus (yes or no); (3) smoking status (none, past, current smoking $<20$ cigarettes/ day or current smoking $\geq 20$ cigarettes/day); (4) alcohol intake (men: none, $<150,150-299,300-449$ or $\geq 450 \mathrm{~g}$ ethanol per week; women: none, $<150,150-299$ or $\geq 300 \mathrm{~g}$ ethanol per week); (5) history of hypertension (yes or no); (6) exercise frequency (less than once/week or once or more times per week); (7) coffee consumption (none, less than daily, 1 cup/day, $\geq 2-3$ cups/day); (8) energy-adjusted magnesium intake (mg; continuous); and (9) total energy intake (kJ; continuous). The above analyses were also performed for selected foods containing high amounts of calcium: total dairy products, milk, cheese and yogurt. The analysis for calcium was stratified according to vitamin D intake (less than median or median or greater) to assess whether vitamin $D$ had a modifying effect on the association between calcium and diabetes risk. Similarly, we examined the above associations according to BMI $(<25$ or $\geq 25 \mathrm{~kg} / \mathrm{m}^{2}$ ), smoking status (non-smokers or current smokers) and drinking status (non-drinkers or current drinkers), since these variables are known to be associated with the risk of type 2 diabetes and might modify nutrientdiabetes associations. An interaction term of two exposure variables was created and added in the model to assess a statistical interaction. Tests for trends were assessed by assigning the median intake value of each quartile of nutrients or food intake in each category. All $p$ values were two sided; statistical significance was determined at $p<0.05$.

\section{Results}

During the 5-year follow-up period, 1,114 participants were newly diagnosed with diabetes (634 men [2.4\%], 480 women [1.4\%]). Table 1 shows the potential confounders according to dietary calcium and vitamin $\mathrm{D}$ intake at baseline. The dietary intake of calcium was positively associated with age and sports participation in both sexes, but was inversely associated with current smoking and heavy alcohol drinking in both sexes, and with BMI and 


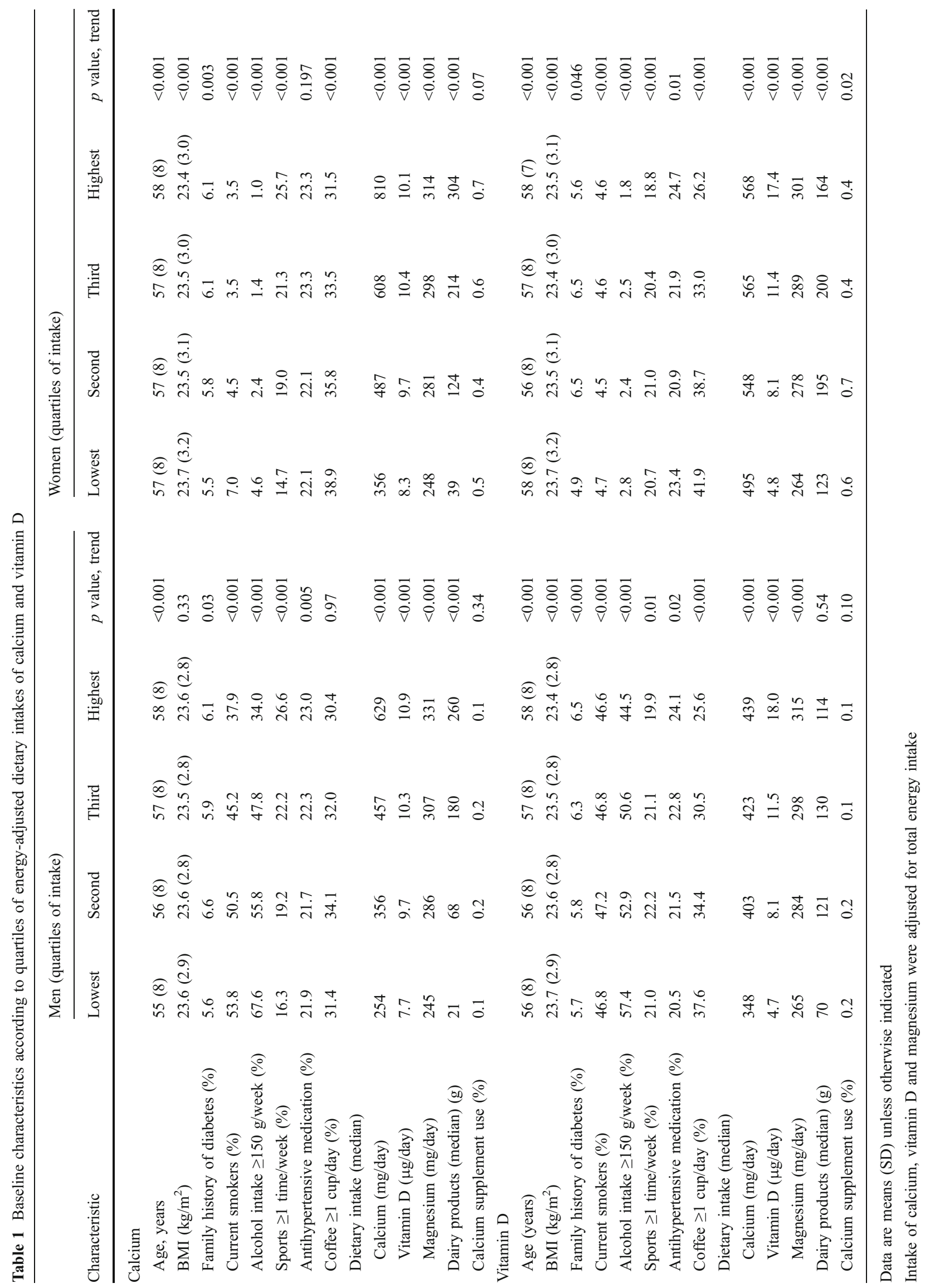


coffee consumption among women. The dietary intake of vitamin D was positively associated with age in both sexes, but was inversely associated with BMI, heavy alcohol intake, sports participation and coffee consumption in both sexes, and with calcium supplement use among women.

In the analysis with adjustments for age and area only, we observed a statistically significant inverse association between dietary calcium intake and the risk of type 2 diabetes in women (Table 2). The odds ratio comparing the highest vs lowest quartile group of calcium intake was 0.74 (95\% CI $0.57-0.96 ; p=0.039$ for trend) among women. The association was slightly attenuated and no longer statistically significant after further adjustment for other potential confounders. The multivariable odds ratio for the highest vs lowest quartiles of calcium intake was $0.76(95 \% \mathrm{CI}$ $0.56-1.03 ; p=0.095$ for trend) among women. The exclusion of calcium supplement users from the analysis $(n=25,836$ for men, $n=33,735$ for women) did not notably alter the results; the odds ratio was 0.77 (95\% CI $0.56-1.04 ; p=0.116$ for trend) among women. There was no association between calcium intake and the risk of diabetes in men. Vitamin D intake alone was not appreciably associated with the risk of type 2 diabetes either in men or in women. Additional adjustment of saturated fat intake did not appreciably alter these results (data not shown).

In women, the intake of dairy foods was significantly inversely associated with the risk of type 2 diabetes. In models with adjustment of age and area only, the odds ratios for the highest vs lowest intake category were 0.65 (95\% CI $0.49-0.88 ; p=0.007$ for trend), 0.79 (95\% CI $0.64-0.97 ; p=0.02$ for trend), 0.94 ( $95 \%$ CI $0.68-1.30 ; p=$ 0.71 for trend) and $0.72(95 \%$ CI $0.55-0.93 ; p=0.04$ for

Table 2 ORs of type 2 diabetes according to quartiles of energy-adjusted dietary intake of calcium and vitamin D

\begin{tabular}{|c|c|c|c|c|}
\hline Intake per quartile and sex & At risk $(n)$ & Cases $(n)$ & $\begin{array}{l}\text { Age- and area-adjusted } \\
\text { OR }(95 \% \mathrm{CI})\end{array}$ & $\begin{array}{l}\text { Multivariable } \\
\text { OR }(95 \% \mathrm{CI})^{*}\end{array}$ \\
\hline \multicolumn{5}{|l|}{ Calcium } \\
\hline \multicolumn{5}{|l|}{ Men } \\
\hline Lowest & 6,469 & 174 & 1.00 & 1.00 \\
\hline Second & 6,469 & 143 & $0.82(0.66-1.03)$ & $0.83(0.66-1.05)$ \\
\hline Third & 6,470 & 162 & $0.93(0.75-1.16)$ & $0.97(0.76-1.24)$ \\
\hline Highest & 6,469 & 155 & $0.89(0.71-1.11)$ & $0.93(0.71-1.22)$ \\
\hline$p$ value for trend & & & 0.52 & 0.95 \\
\hline \multicolumn{5}{|l|}{ Women } \\
\hline Lowest & 8,479 & 134 & 1.00 & 1.00 \\
\hline Second & 8,480 & 118 & $0.88(0.69-1.14)$ & $0.90(0.70-1.18)$ \\
\hline Third & 8,480 & 126 & $0.94(0.73-1.20)$ & $0.95(0.73-1.25)$ \\
\hline Highest & 8,480 & 102 & $0.74(0.57-0.96)$ & $0.76(0.56-1.03)$ \\
\hline$p$ value for trend & & & 0.039 & 0.095 \\
\hline \multicolumn{5}{|l|}{ Vitamin D } \\
\hline \multicolumn{5}{|l|}{ Men } \\
\hline Lowest & 6,469 & 156 & 1.00 & 1.00 \\
\hline Second & 6,469 & 182 & $1.20(0.96-1.49)$ & $1.17(0.93-1.46)$ \\
\hline Third & 6,470 & 145 & $0.96(0.76-1.21)$ & $0.93(0.73-1.18)$ \\
\hline Highest & 6,469 & 151 & $1.01(0.79-1.28)$ & $0.96(0.74-1.23)$ \\
\hline$p$ value for trend & & & 0.58 & 0.35 \\
\hline \multicolumn{5}{|l|}{ Women } \\
\hline Lowest & 8,479 & 139 & 1.00 & 1.00 \\
\hline Second & 8,480 & 104 & $0.76(0.58-0.98)$ & $0.77(0.59-1.00)$ \\
\hline Third & 8,480 & 105 & $0.76(0.58-0.99)$ & $0.76(0.57-1.00)$ \\
\hline Highest & 8,480 & 132 & $0.94(0.72-1.23)$ & $0.88(0.67-1.16)$ \\
\hline$p$ value for trend & & & 0.94 & 0.67 \\
\hline
\end{tabular}

*Adjusted further for age (continuous), area (nine Public Health Center areas), BMI $\left(<21,21-22.9,23-24.9,25-26.9\right.$ or $\geq 27 \mathrm{~kg} / \mathrm{m}^{2}$ ), family history of diabetes mellitus (yes or no), smoking status (none, past, current smoking $<20$ or $\geq 20$ cigarettes/day), alcohol intake (men: none, $<150$, 150-299, 300-449 or $\geq 450 \mathrm{~g}$ ethanol/week; women: none, $<150,150-299$ or $\geq 300 \mathrm{~g}$ ethanol/week), history of hypertension (yes or no), exercise frequency (less than once/week or $\geq$ once/week), consumption of coffee (less than daily, $1-3$ cups/day or $\geq 4$ cups/day), energy-adjusted magnesium (continuous) and total energy (continuous) 
trend) for total dairy products, milk, cheese and yogurt, respectively. In multivariable analyses, these associations were attenuated. No significant association between dairy product intake and the risk of diabetes was observed in men (Table 3). We repeated the above analyses without adjustment for intake of magnesium, a component of dairy foods, but the results were not materially changed (data not shown).

In a stratified analysis according to vitamin $\mathrm{D}$ intake, the inverse association between calcium intake and the risk of diabetes was more pronounced among participants who consumed a median or greater amount of vitamin D; the odds ratios $(95 \% \mathrm{CI})$ for the highest vs lowest quartiles of calcium intake were $0.62(0.41-0.94 ; p=0.050$ for trend $)$ in men and $0.59(0.38-0.91 ; p=0.043$ for trend) in women (Table 4). In contrast, calcium intake was not associated with the risk of diabetes in either sex in the lower vitamin D intake group. $p$ for interaction between calcium (continuous) and vitamin D (dichotomous) was 0.02 and 0.06 for men and women, respectively. In stratified analyses according to BMI, smoking status or alcohol intake, the associations with calcium or vitamin D were not notably different between the stratified subgroups (data not shown).

\section{Discussion}

In this large-scale cohort of Japanese adults, intake of calcium and vitamin $\mathrm{D}$ was not associated with a significantly lower risk of type 2 diabetes. However, both in men and women there was a clear decreasing trend of type 2 diabetes risk with increasing dietary intake of calcium among persons with a higher vitamin D intake. Dairy food intake was inversely associated with the risk of type 2 diabetes in women, but not in men. To our knowledge, this is the first prospective study in a Japanese population to show an association between both calcium and vitamin $\mathrm{D}$ intake and the risk of type 2 diabetes.

Although calcium intake was not clearly associated with risk of type 2 diabetes, there was a suggestion of an inverse association in women. This finding appears to be consistent with results from studies in US American women showing a marginally significant inverse association with dietary calcium intake in multivariable analyses $[7,8]$. A notable finding in our study is that a distinct inverse association between calcium and the risk of diabetes was observed in the higher, but not in the lower vitamin D intake subgroup.

Table 3 ORs of type 2 diabetes according to the intake of dairy products, milk, cheese and yogurt

\begin{tabular}{|c|c|c|c|c|c|c|}
\hline \multirow[b]{2}{*}{ Intake (g/day) per product group } & \multicolumn{3}{|l|}{ Men } & \multicolumn{3}{|l|}{ Women } \\
\hline & At risk $(n)$ & Cases $(n)$ & $\begin{array}{l}\text { Multivariable } \\
\text { OR }(95 \% \mathrm{CI})^{*}\end{array}$ & At risk $(n)$ & Cases $(n)$ & $\begin{array}{l}\text { Multivariable } \\
\text { OR }(95 \% \mathrm{CI})^{*}\end{array}$ \\
\hline \multicolumn{7}{|l|}{ Dairy products } \\
\hline$<50$ & 8,776 & 217 & 1.00 & 7,674 & 136 & 1.00 \\
\hline $50-<150$ & 6,016 & 141 & $0.99(0.79-1.23)$ & 8,154 & 113 & $0.82(0.64-1.07)$ \\
\hline $150-<300$ & 7,772 & 189 & $1.04(0.85-1.28)$ & 11,958 & 160 & $0.82(0.64-1.04)$ \\
\hline$\geq 300$ & 3,313 & 87 & $1.18(0.90-1.56)$ & 6,133 & 71 & $0.71(0.51-0.98)$ \\
\hline$p$ value for trend & & & 0.21 & & & 0.054 \\
\hline \multicolumn{7}{|l|}{ Milk } \\
\hline$<50$ & 12,102 & 304 & 1.00 & 12,485 & 191 & 1.00 \\
\hline $50-<100$ & 732 & 19 & $1.07(0.66-1.72)$ & 1,282 & 23 & $1.29(0.83-2.01)$ \\
\hline $100-<200$ & 4,463 & 98 & $0.90(0.71-1.13)$ & 6,349 & 98 & $1.08(0.84-1.39)$ \\
\hline$\geq 200$ & 8,580 & 213 & $1.02(0.85-1.24)$ & 13,803 & 168 & $0.87(0.70-1.09)$ \\
\hline$p$ value for trend & & & 0.88 & & & 0.16 \\
\hline \multicolumn{7}{|l|}{ Cheese } \\
\hline 0 & 13,085 & 326 & 1.00 & 16,939 & 253 & 1.00 \\
\hline $0.1-<5$ & 10,390 & 261 & $1.08(0.91-1.29)$ & 13,570 & 182 & $1.08(0.88-1.33)$ \\
\hline$\geq 5$ & 2,402 & 47 & $0.88(0.64-1.21)$ & 3,410 & 45 & $1.12(0.80-1.57)$ \\
\hline$p$ value for trend & & & 0.39 & & & 0.56 \\
\hline \multicolumn{7}{|l|}{ Yogurt } \\
\hline 0 & 15,820 & 386 & 1.00 & 12,551 & 209 & 1.00 \\
\hline $0.1-<60$ & 7,528 & 193 & $1.14(0.95-1.37)$ & 14,100 & 186 & $0.85(0.69-1.05)$ \\
\hline$\geq 60$ & 2,529 & 55 & $1.01(0.75-1.36)$ & 7,268 & 85 & $0.77(0.58-1.01)$ \\
\hline$p$ value for trend & & & 0.94 & & & 0.13 \\
\hline
\end{tabular}

*Adjusted variables as in Table 2 (footnote) 
Table 4 Multivariable odds ratios of type 2 diabetes according to quartiles of calcium intake, stratified by median intake of vitamin D

\begin{tabular}{|c|c|c|c|c|c|c|}
\hline \multirow[b]{2}{*}{$\begin{array}{l}\text { Quartiles of calcium intake } \\
\text { per vitamin D intake }\end{array}$} & \multicolumn{3}{|l|}{ Men } & \multicolumn{3}{|l|}{ Women } \\
\hline & At risk $(n)$ & Cases $(n)$ & $\begin{array}{l}\text { Multivariable } \\
\text { OR }(95 \% \mathrm{CI}) *\end{array}$ & At risk $(n)$ & Cases $(n)$ & $\begin{array}{l}\text { Multivariable } \\
\text { OR }(95 \% \mathrm{CI})^{*}\end{array}$ \\
\hline \multicolumn{7}{|l|}{ Low vitamin $\mathrm{D}(<$ median $)$} \\
\hline Lowest & 4,166 & 107 & 1.00 & 5,053 & 73 & 1.00 \\
\hline Second & 3,218 & 71 & $0.86(0.63-1.19)$ & 4,195 & 63 & $1.14(0.79-1.63)$ \\
\hline Third & 2,870 & 73 & $1.01(0.72-1.41)$ & 3,762 & 56 & $1.12(0.76-1.66)$ \\
\hline Highest & 2,684 & 87 & $1.31(0.92-1.89)$ & 3,949 & 51 & $0.94(1.62-1.43)$ \\
\hline$p$ value for trend & & & 0.08 & & & 0.70 \\
\hline \multicolumn{7}{|l|}{ High vitamin $\mathrm{D}$ ( $\geq$ median) } \\
\hline Lowest & 2,303 & 67 & 1.00 & 3,426 & 61 & 1.00 \\
\hline Second & 3,251 & 72 & $0.76(0.53-1.08)$ & 4,285 & 55 & $0.70(0.48-1.02)$ \\
\hline Third & 3,600 & 89 & $0.87(0.61-1.25)$ & 4,718 & 70 & $0.79(0.54-1.15)$ \\
\hline Highest & 3,785 & 68 & $0.62(0.41-0.94)$ & 4,531 & 51 & $0.59(0.38-0.91)$ \\
\hline$p$ value for trend & & & 0.050 & & & 0.043 \\
\hline
\end{tabular}

*Adjustment variables, see Table 2 (footnote)

$p=0.02$ and $p=0.06$ for interaction of calcium (continuous) and vitamin D (dichotomous), men and women respectively

This is in line with the hypothesis that calcium and vitamin D act jointly to protect against type 2 diabetes. In a prospective study among US American nurses [7], a combined daily intake of $>1,200 \mathrm{mg}$ of calcium and $>800$ IU of vitamin D was associated a $33 \%$ lower risk of type 2 diabetes compared with a daily intake of $\leq 600 \mathrm{mg}$ and $\leq 400 \mathrm{IU}$ respectively. Although the lowest risk was observed in persons with a combined high intake of calcium and vitamin $\mathrm{D}$, the benefit of the two nutrients appears to be additive in the US study.

We found a marginally significant risk reduction associated with the highest intake of calcium and dairy products in women, but not in men. This sex-related difference in association could be ascribed to chance, but another explanation is possible. Thus in the present cohort, women consumed greater amounts of calcium and dairy products than men, with median daily calcium intake at $404 \mathrm{mg}$ for men and $546 \mathrm{mg}$ for women and that for dairy products at $111 \mathrm{~g}$ for men and $171 \mathrm{~g}$ for women. If apparent risk reduction is observed only above a certain intake level, comparatively lower intake of calcium and dairy products in men than in women could explain the observed discrepancy in association.

A few intervention studies have examined the effects of the combined intake of calcium and vitamin D on type 2 diabetes, but their results have been inconsistent. Specifically, supplementation with $400 \mathrm{IU}$ of vitamin D and 1,000 $\mathrm{mg}$ of calcium did not reduce the risk of type 2 diabetes over a 7 -year followup among participants with normal and impaired fasting glucose levels [13]. In another trial of 221 elderly persons with normal glucose tolerance, combined supplementation with $700 \mathrm{IU}$ of vitamin D and $500 \mathrm{mg}$ of calcium citrate malate had no effect on glycaemia or insulin resistance during a 3-year follow-up, compared with the placebo group [20]. In that study, however, the administration of calcium and vitamin D supplements significantly attenuated the increase in fasting glycaemia and insulin resistance among participants with impaired fasting glucose, compared with control participants [20].

Regarding the intake of dairy foods, we found statistically significant inverse associations with the risk of type 2 diabetes in age- and area-adjusted analyses in women, but the association was attenuated after multivariable adjustments. An inverse association with type 2 diabetes has been reported for the intake of dairy foods in men [9] and women [7], for the intake of low-fat dairy foods in women $[8,10]$ and for yogurt intake in men [9] and women [10]. Our study did not find a risk reduction associated with milk intake, a finding consistent with the results of American studies [9, 10], although one study [9] reported a lower risk of type 2 diabetes with low-fat milk intake.

Vitamin D intake was not independently associated with the risk of type 2 diabetes in our study. Two prospective studies have shown an inverse association with supplemental [7] and total [11] vitamin D intake, but not with dietary vitamin D intake. A possible explanation for the lack of association with dietary vitamin D in our study and in others is the fact that sunlight-induced cutaneous synthesis of vitamin D also contributes to systemic vitamin D levels [4]. Consequently, dietary vitamin D intake alone may not explain the overall vitamin D status. Data from the present study population do not enable us to examine the 
contribution of dietary vitamin D intake to circulating 25-OHD concentrations, an indicator of systemic vitamin D status. However, a study of Japanese women, which was conducted in winter [21], showed that persons who frequently consumed fish, a rich source of vitamin D, had higher mean blood 25-OHD concentrations than those who consumed fish infrequently. Thus, dietary vitamin D intake could be used in ranking systemic vitamin D status at least when the amount of sunlight-induced cutaneous synthesis of vitamin D is low.

The precise mechanisms whereby calcium and vitamin D exert glucose-lowering effects are not clear. Calcium is essential for insulin-mediated intracellular processes. Intracellular calcium levels are tightly controlled within a narrow range to maintain insulin signalling [22]. Calcium deficiency leads to the secretion of parathyroid hormone and increases calcium inflow from the extracellular fluid into intracellular regions, resulting in cellular calcium overload and impaired insulin sensitivity [5, 23]. In epidemiological studies, calcium intake was positively associated with insulin sensitivity [24-26]. Vitamin D is also involved in insulin regulation [3, 4]. 1,25Dihydroxyvitamin $\mathrm{D}_{3}$, an active form of circulating vitamin $\mathrm{D}$, binds to the vitamin $\mathrm{D}$ receptor on pancreas beta cells and enhances insulin receptor expression, resulting in improved insulin sensitivity [27, 28]. Vitamin D deficiency has also been shown to impair insulin secretion in experimental studies [3, 4]. Moreover, because vitamin D facilitates calcium absorption in the intestines [29], vitamin D and calcium may act synergistically to reduce the risk of type 2 diabetes. Dairy foods rich in calcium may decrease the risk of diabetes through calcium-related mechanisms. In addition, milk protein induces the release of insulinogenic amino acids and the peptide hormone incretin, both of which augment insulin secretion [30].

Japanese patients with type 2 diabetes are on average leaner than white counterparts, which might reflect differences in insulin secretion and sensitivity between the two ethnic groups [31]. A multi-ethnic study of US American women [32] found that Japanese-Americans had lower beta cell function than non-Hispanic whites, suggesting a need to improve beta cell function as well as insulin sensitivity in order to prevent type 2 diabetes in the Japanese. If calcium and vitamin D have beneficial effects on beta cell function and insulin sensitivity, sufficient intake of these nutrients may have a large impact on reducing the risk of type 2 diabetes among Japanese.

The major strengths of our study include its prospective design, large sample size and the use of a validated food frequency questionnaire. However, several study limitations should also be mentioned. First, the incidence of diabetes was ascertained on the basis of self-reported information obtained from the participants. According to a validation study, self-reported diabetes exhibited a fairly good agreement with documented diabetes on the basis of medical records $(94 \%)$, while sensitivity $(82.6 \%)$ and specificity (99.7\%) of self-reported diabetes were also high. Second, the dietary intake of calcium and vitamin D was only measured at one time-point and thus may not reflect long-term exposure. Third, we were unable to distinguish regular from low-fat dairy foods, two categories that may have different effects on the risk of diabetes. Fourth, we did not consider sunlight-induced cutaneous synthesis of vitamin D. Studies involving the measurement of blood 25-OHD, a marker of systemic vitamin D exposure, could reveal the association between vitamin $\mathrm{D}$ and the risk of type 2 diabetes more precisely. Finally, significant results obtained in subgroup analysis may be due to chance and thus should be interpreted with caution.

In conclusion, the present study provided no clear evidence to support an independent role of calcium and vitamin $\mathrm{D}$ in the development of type 2 diabetes. An inverse association between calcium intake and type 2 diabetes risk in persons with a higher vitamin $\mathrm{D}$ intake suggests that these nutrients may act jointly, rather than independently, in lowering risk of type 2 diabetes. This possibility warrants further investigation.

Acknowledgements The authors thank all the staff members in each study area and in the central office for their painstaking efforts in conducting the baseline and follow-up research. We thank Y. Matsushita and A. Nanri (both International Medical Center of Japan) for their helpful discussions, and K. Saito (International Medical Center of Japan) for editing the manuscript. This study was supported by Grants-in-aid for Cancer Research (19shi-2) and a Health Sciences Research Grant (Research on Comprehensive Research on Cardiovascular Diseases H19-016) from the Ministry of Health, Labour and Welfare of Japan.

Duality of interest The authors declare that there is no duality of interest associated with this manuscript.

\section{References}

1. Wild S, Roglic G, Green A, Sicree R, King H (2004) Global prevalence of diabetes: estimates for the year 2000 and projections for 2030. Diabetes Care 27:1047-1053

2. Iso $H$ (2008) Changes in coronary heart disease risk among Japanese. Circulation 118:2725-2729

3. Palomer X, Gonzalez-Clemente JM, Blanco-Vaca F, Mauricio D (2008) Role of vitamin D in the pathogenesis of type 2 diabetes mellitus. Diabetes Obes Metab 10:185-197

4. Tai K, Need AG, Horowitz M, Chapman IM (2008) Vitamin D, glucose, insulin, and insulin sensitivity. Nutrition 24:279-285

5. Zemel MB (1998) Nutritional and endocrine modulation of intracellular calcium: implications in obesity, insulin resistance and hypertension. Mol Cell Biochem 188:129-136

6. Pittas AG, Lau J, Hu FB, Dawson-Hughes B (2007) The role of vitamin $\mathrm{D}$ and calcium in type 2 diabetes. A systematic review and meta-analysis. J Clin Endocrinol Metab 92:2017-2029 
7. Pittas AG, Dawson-Hughes B, Li T et al (2006) Vitamin D and calcium intake in relation to type 2 diabetes in women. Diabetes Care 29:650-656

8. van Dam RM, Hu FB, Rosenberg L, Krishnan S, Palmer JR (2006) Dietary calcium and magnesium, major food sources, and risk of type 2 diabetes in U.S. black women. Diabetes Care 29:2238-2243

9. Choi HK, Willett WC, Stampfer MJ, Rimm E, Hu FB (2005) Dairy consumption and risk of type 2 diabetes mellitus in men: a prospective study. Arch Intern Med 165:997-1003

10. Liu S, Choi HK, Ford E et al (2006) A prospective study of dairy intake and the risk of type 2 diabetes in women. Diabetes Care 29:1579-1584

11. Liu S, Song Y, Ford ES, Manson JE, Buring JE, Ridker PM (2005) Dietary calcium, vitamin D, and the prevalence of metabolic syndrome in middle-aged and older U.S. women. Diabetes Care 28:2926-2932

12. Mattila C, Knekt P, Mannisto S et al (2007) Serum 25hydroxyvitamin D concentration and subsequent risk of type 2 diabetes. Diabetes Care 30:2569-2570

13. de Boer IH, Tinker LF, Connelly S et al (2008) Calcium plus vitamin D supplementation and the risk of incident diabetes in the Women's Health Initiative. Diabetes Care 31:701-707

14. The Study Circle for Health and Nutrition Information (2009) The National Health and Nutrition Survey in Japan 2006. DaiichiShuppan, Tokyo (in Japanese)

15. Tsugane S, Sobue T (2001) Baseline survey of JPHC studydesign and participation rate. Japan Public Health Center-based Prospective Study on Cancer and Cardiovascular Diseases. J Epidemiol 11(Suppl):S24-S29

16. Ishihara J, Inoue M, Kobayashi $M$ et al (2006) Impact of the revision of a nutrient database on the validity of a selfadministered food frequency questionnaire (FFQ). J Epidemiol 16:107-116

17. Waki K, Noda M, Sasaki S et al (2005) Alcohol consumption and other risk factors for self-reported diabetes among middle-aged Japanese: a population-based prospective study in the JPHC study cohort I. Diabetes Med 22:323-331

18. Kosaka K (1994) Diagnostic criteria for diabetes mellitus in Japan-from a report of the Japan Diabetes Society (JDS) Committee on the Diagnosis of Diabetes Mellitus, 1982. Diabetes Res Clin Pract 24(Suppl):S59-S62

19. Willett W, Stampfer MJ (1986) Total energy intake: implications for epidemiologic analyses. Am J Epidemiol 124:17-27

20. Pittas AG, Harris SS, Stark PC, Dawson-Hughes B (2007) The effects of calcium and vitamin D supplementation on blood glucose and markers of inflammation in nondiabetic adults. Diabetes Care 30:980-986

21. Nakamura K, Nashimoto M, Hori Y, Yamamoto M (2000) Serum 25-hydroxyvitamin $\mathrm{D}$ concentrations and related dietary factors in peri- and postmenopausal Japanese women. Am J Clin Nutr $71: 1161-1165$

22. Draznin B, Sussman K, Kao M, Lewis D, Sherman N (1987) The existence of an optimal range of cytosolic free calcium for insulinstimulated glucose transport in rat adipocytes. J Biol Chem 262:14385-14388

23. Fujita T, Palmieri GM (2000) Calcium paradox disease: calcium deficiency prompting secondary hyperparathyroidism and cellular calcium overload. J Bone Miner Metab 18:109-125

24. Drouillet P, Balkau B, Charles MA et al (2007) Calcium consumption and insulin resistance syndrome parameters. Data from the Epidemiological Study on the Insulin Resistance Syndrome (DESIR). Nutr Metab Cardiovasc Dis 17:486-492

25. Ma B, Lawson AB, Liese AD, Bell RA, Mayer-Davis EJ (2006) Dairy, magnesium, and calcium intake in relation to insulin sensitivity: approaches to modeling a dose-dependent association. Am J Epidemiol 164:449-458

26. dos Santos LC, de Padua Cintra I, Fisberg M, Martini LA (2008) Calcium intake and its relationship with adiposity and insulin resistance in post-pubertal adolescents. J Hum Nutr Diet 21:109-116

27. Calle C, Maestro B, Garcia-Arencibia M (2008) Genomic actions of 1,25-dihydroxyvitamin D3 on insulin receptor gene expression, insulin receptor number and insulin activity in the kidney, liver and adipose tissue of streptozotocin-induced diabetic rats. BMC Mol Biol 9:65

28. Maestro B, Campion J, Davila N, Calle C (2000) Stimulation by 1,25-dihydroxyvitamin D3 of insulin receptor expression and insulin responsiveness for glucose transport in U-937 human promonocytic cells. Endocr J 47:383-391

29. Khanal RC, Nemere I (2008) Regulation of intestinal calcium transport. Annu Rev Nutr 28:179-196

30. Nilsson M, Stenberg M, Frid AH, Holst JJ, Bjorck IM (2004) Glycemia and insulinemia in healthy subjects after lactoseequivalent meals of milk and other food proteins: the role of plasma amino acids and incretins. Am J Clin Nutr 80:1246-1253

31. Sone H, Ito H, Ohashi Y, Akanuma Y, Yamada N (2003) Obesity and type 2 diabetes in Japanese patients. Lancet 361:85

32. Torréns JI, Skurnick J, Davidow AL et al (2004) Ethnic differences in insulin sensitivity and beta-cell function in premenopausal or early perimenopausal women without diabetes: the Study of Women's Health Across the Nation (SWAN). Diabetes Care 27:354-361 